\title{
Acute Kidney Injury in Pediatric Patients Treated with Vancomycin and Piperacillin-Tazobactam Versus Vancomycin and Cefotaxime: A Single- center Study
}

\author{
Rewaa Alqurashi ${ }^{1}$, Mawaddah Batwa ${ }^{1}$, Bashayer Alghamdi ${ }^{1}$, Saja Aljohani ${ }^{1}$, Nada Zaher ${ }^{1}$, Amal \\ Alzahrani $^{1}$, Eman Aldigs ${ }^{2}$, Osama Safdar ${ }^{3}$ \\ 1. Internal Medicine, King Abdulaziz University, Jeddah, SAU 2. Medical Microbiology and Parasitology, King Abdulaziz \\ University, Jeddah, SAU 3. Pediatrics, King Abdulaziz University, Jeddah, SAU
}

Corresponding author: Rewaa Alqurashi, alqurashirewaa@gmail.com

\begin{abstract}
Background

Previous literature showed a higher incidence of acute kidney injury (AKI) in pediatric patients using vancomycin + piperacillin-tazobactam compared to cefepime + vancomycin. Our aim was to compare the incidence of developing AKI during the use of vancomycin + cefotaxime with that during the use of vancomycin + piperacillin-tazobactam in pediatric patients.
\end{abstract}

Methods

This was a retrospective, matched cohort study that used electronic records from May 1, 2015 through April 30, 2018 for all patients aged less than 16 years who received intravenous (IV) vancomycin + piperacillintazobactam or cefotaxime + vancomycin for at least 72 hours. AKI was defined by Kidney Disease Improving Global Outcomes (KDIGO) guidelines. Each patient from the vancomycin + piperacillin-tazobactam group was matched 1:1 with those in the vancomycin + cefotaxime group according to their age, chronic disease, gender, and the number of concomitant nephrotoxic agents. A total of 64 cases were included. Statistical analysis was performed using descriptive statistics and binary logistic regression.

Results

AKI developed in 10 of 32 patients (31.25\%) who were using vancomycin + piperacillin-tazobactam. On the other hand, 13 of 32 patients $(40.62 \%)$ were using cefotaxime + vancomycin $(p=0.047)$. Of the 10 patients who were on vancomycin + piperacillin-tazobactam regimen, $80 \%$ developed AKI Stage I. Of the 13 patients who were using cefotaxime + vancomycin, $46 \%$ developed AKI Stage II, although no statistical significance was noted in all stages.

Received 01/15/2020

Review began 01/16/2020

Review ended 01/16/2020

Published 01/28/2020

๑) Copyright 2020

Alqurashi et al. This is an open access article distributed under the terms of the Creative Commons Attribution License CC-BY 3.0., which permits unrestricted use, distribution, and reproduction in any medium, provided the original author and source are credited.
Conclusion

Our study showed that patients treated with cefotaxime and vancomycin showed a higher incidence of AKI than patients treated with vancomycin and piperacillin-tazobactam, although the study showed no statistical significance.

Categories: Pediatrics, Infectious Disease, Nephrology

Keywords: infectious diseases, antibiotics, pediatrics, nephrology

\section{Introduction}

Drug exposure plays a significant role in the destruction of kidney function. Since kidneys are mainly responsible for maintaining homeostasis in the body and eliminating waste products, they are more susceptible to xenobiotics [1]. Acute kidney injury (AKI) is characterized by an abrupt decrease in kidney function within hours due to structural damage. AKI has worse outcomes in hospitalized patients [2]. Its definition has evolved from the RIFLE (Risk, Injury, Failure, Loss of Kidney Function, and End-stage Kidney Disease) criteria and the AKIN (Acute Kidney Injury Network) classification to the Kidney Disease Improving Global Outcomes (KDIGO) classification in 2012 [3-5]. KDIGO defines AKI on the basis of serum creatinine and urine output over a specific duration. Unlike the RIFLE criteria, which included glomerular filtration rate (GFR) as an important parameter, clinical judgment was required for patients to meet the AKI, RIFLE, and AKIN criteria, which were originally developed for average-sized adults and not for children [6].

Vancomycin (VAN) is a common nephrotoxic antibiotic, which has multiple FDA-approved and off-label 
clinical uses [7]. It was reported that $10 \%-20 \%$ and $30 \%-40 \%$ of patients had AKI caused by conventional and high doses of VAN therapy, respectively. The increased production of reactive oxygen species during oxidative stress was thought to be the most probable mechanism for its nephrotoxicity [7]. There are numerous documented risk factors that could potentiate the occurrence of VAN-induced nephrotoxicity. These risk factors include increased VAN levels ( $>20 \mathrm{mg} / \mathrm{L}$ ), administering high doses to patients ( $>4 \mathrm{~g} /$ day), prolonged therapy ( $>7$ days), intensive care unit admittance (especially if it was a prolonged stay), and concomitant treatment with nephrotoxic agents, such as nonsteroidal anti-inflammatory drugs, loop diuretics, and more $[2,8]$.

Patients with suspected sepsis are at higher risk of developing drug-associated adverse effects due to the use of multiple antibiotic combinations (sepsis development in patients using VAN alone was $13.1 \%$ vs $27.2 \%$ in combination groups) [9-10]. In children under antibiotic treatment, the major adverse drug reactions were caused by $\beta$-lactams and VAN [11]. Piperacillin-tazobactam (PTZ) is an antipseudomonal antibiotic that inhibits $\beta$-lactam/ $\beta$-lactamase and is used in combination with VAN for hospitalized patients [12]. Furthermore, cefotaxime is a third-generation cephalosporin that acts against gram-negative and some gram-positive bacteria. It is well distributed throughout the body and is excreted mainly unchanged in the urine [13]. Cefotaxime is used in combination with VAN to treat serious systemic infections (osteomyelitis, infective endocarditis, skin and soft tissue infection, and many others) [14].

Several studies have reported a higher incidence of AKI in adult patients using VAN + PTZ (VAN-PTZ) combination (16.3\% to $34.8 \%$ ) compared to VAN + cefepime, a fourth-generation cephalosporin $(12.5 \%$ to 13.3\%) [10, 15-17]. Similarly, higher rates of nephrotoxicity were observed among patients treated with VAN-PTZ compared with VAN treatment alone; however, the exact mechanism for the increased nephrotoxicity by this combination was not fully understood [18]. Moreover, a robust retrospective cohort study reported a higher incidence of AKI in pediatric patients using VAN-PTZ compared to VAN-cefepime [19].

However, there are no data regarding the incidence of AKI when cefotaxime is used in combination with VAN. Therefore, we tested the safety of VAN + cefotaxime compared to that of VAN-PTZ in a pediatric population. At the King Abdulaziz University Hospital, we prefer using cefotaxime over cefepime to avoid future cefepime resistance.

\section{Materials And Methods Study design}

This study was approved by the Institutional Review Board (IRB) of the King Abdulaziz University Hospital (KAUH) Jeddah, Saudi Arabia. The data were collected from the pediatric system database, Phoenix Data Systems Asset Information Management System (Phoenix Data Systems, Southfield, MI), starting from May 1, 2015 to April 30, 2018. There were 1,129 medical file records of patients who had received IV VAN out of which we had to extract the data that matched our inclusion criteria.

\section{Study subjects}

Electronic records of all patients aged less than 16 years who received IV VAN-PTZ or IV VAN + cefotaxime for at least 72 hours were reviewed; baseline serum creatinine (measured within a week before antibiotic administration), plus daily creatinine levels during the antibiotic course were monitored. AKI was defined as an increase of serum creatinine ( $\mathrm{SCr}$ ) of $>0.3 \mathrm{mg} / \mathrm{dl}$ or an increase in $\mathrm{SCr}$ to $\geqslant 1.5$ times baseline. If any patient met the criteria, he or she was defined to have AKI Stage 1, which was defined as a 1.5 to 1.9 -fold increase in SCr from the baseline or SCr $\geqslant 0.3 \mathrm{mg} / \mathrm{dL}(\geqslant 26.5 \mu \mathrm{mol} / \mathrm{L}$ ). Stage 2 was defined as a 2.0 to 2.9 -fold increase in $\mathrm{SCr}$ from the baseline, while Stage 3 was defined as three-fold higher $\mathrm{SCr}$ or $\mathrm{SCr} \geqslant 4.0 \mathrm{mg} / \mathrm{dl}$ $(\geqslant 353.6 \mu \mathrm{mol} / \mathrm{L})$.

Subject characteristics were also obtained from the records including age, gender, weight, height, chronic disease, indication for antibiotics, nephrotoxic agents, VAN trough (highest measurement that was taken in a steady-state prior to AKI development), therapy duration (at least one dose on a given day), and admittance to the intensive care unit (ICU).

Each patient from the VAN-PTZ group was matched 1:1 with those in the VAN-cefotaxime group according to their age (younger than three months, equal or between three months and one year, equal to or older than one year), chronic disease (congenital heart disease, malignancy, and others), gender, and the number of concomitant nephrotoxic agents.

\section{Exclusion criteria}

Patients with underlying kidney disease (preexisting renal dysfunction, structural kidney disease, undergoing dialysis, or AKI at admission) or did not have a match or who took oral VAN were excluded from the study (only patients who take IV antibiotics can be tracked properly in our hospital system). All patients who did not have a match in the other group were excluded from the study. 


\section{Cureus}

\section{Statistical analysis}

The collected data were entered using an online Google ${ }^{\circledR}$ drive form and transferred to Microsoft ${ }^{\circledR}$ Excel. Our statistical analysis was performed using the IBM ${ }^{\circledR}$ Statistical Package for Social Sciences (SPSS), v. 23 software package (IBM SPSS Statistics, Armonk, NY). The incidence of developing AKI that was caused by VAN-PTZ or VAN + cefotaxime was calculated using bivariate logistic regression. Binary logistic regression was used for all variables (age, gender, weight, chronic disease, indication for antibiotics, nephrotoxic agents, VAN trough, therapy duration, and ICU admission) to determine their association with the incidence of developing AKI. We considered p-value $<0.05$ as significant. All variables in each treatment group and AKI development were measured using descriptive statistics.

\section{Results}

One-hundred and sixty-two patients were eligible for inclusion and 93 patients were excluded from the study because no match was found in the other treatment group. Five patients were from the Tazocin - vancomycin group and were excluded because there was no match for them in the other treatment group. A total of 64 cases were included: ( $\mathrm{n}=32$ for VAN-PTZ) and $(\mathrm{n}=32$ for VAN + cefotaxime).

\begin{tabular}{|c|c|c|c|}
\hline Characteristics & VAN-PTZ $(n=32)^{a}$ & VAN-cefotaxime $(n=32)^{a}$ & P-value \\
\hline Age (years) & $1.33( \pm 1.86)$ & $1.29( \pm 1.99)$ & $0.492^{b}$ \\
\hline Male & $18(56.3 \%)$ & $18(56.3 \%)$ & \multirow{2}{*}{$0.126^{b}$} \\
\hline Female & $14(43.8 \%)$ & $14(43.8 \%)$ & \\
\hline Weight & $8.28( \pm 6.70)$ & $7.96( \pm 8.46)$ & $0.619^{b}$ \\
\hline Baseline serum creatinine (mg/dl) & $0.3315( \pm 0.17)$ & $0.47( \pm 0.29)$ & $0.065^{b}$ \\
\hline CHD & $19(59.4 \%)$ & $19(59.4 \%)$ & $0.855^{\mathrm{b}}$ \\
\hline Malignancy & $1(3.1 \%)$ & $1(3.1 \%)$ & $0.678^{b}$ \\
\hline Hypertension & $1(3.1 \%)$ & 4 (12.5\%) & $0.260^{\mathrm{b}}$ \\
\hline Hypotension & $0(0 \%)$ & $1(3.1 \%)$ & $1.000^{\mathrm{b}}$ \\
\hline Primary immunodeficiency & $1(3.1 \%)$ & $0(0 \%)$ & $1.000^{\mathrm{b}}$ \\
\hline Hypoalbuminemia & $1(3.1 \%)$ & $0(0 \%)$ & $1.000^{\mathrm{b}}$ \\
\hline Others & $0(0 \%)$ & $0(0 \%)$ & $1.000^{\mathrm{b}}$ \\
\hline \multicolumn{4}{|l|}{ Indication for antibiotics } \\
\hline Empiric therapy & $9(28.1 \%)$ & $6(18.8 \%)$ & $0.115^{\mathrm{b}}$ \\
\hline Prophylaxis & $4(12.5 \%)$ & $7(21.9 \%)$ & $0.513^{b}$ \\
\hline CNS disease & $1(3.1 \%)$ & $1(3.1 \%)$ & $0.999^{b}$ \\
\hline Bacteremia & $3(9.4 \%)$ & $1(3.1 \%)$ & $0.641^{b}$ \\
\hline Cardiovascular disease & $7(21.9 \%)$ & $10(31.3 \%)$ & $0.600^{b}$ \\
\hline Respiratory disease & $11(34.4 \%)$ & $8(25 \%)$ & $0.505^{b}$ \\
\hline GU/GI disease & $2(6.3 \%)$ & $2(6.3 \%)$ & $0.641^{b}$ \\
\hline SSTI & $1(3.1 \%)$ & $1(3.1 \%)$ & $0.678^{b}$ \\
\hline Febrile neutropenia & $1(3.1 \%)$ & $0(0 \%)$ & $1.000^{\mathrm{b}}$ \\
\hline Sepsis & $12(37.5 \%)$ & $11(34.4 \%)$ & $0.141^{b}$ \\
\hline Therapy duration (days) & $8.47( \pm 3.98)$ & $6.50( \pm 2.83)$ & $0.600^{\mathrm{b}}$ \\
\hline Admittance to ICU & $16(50 \%)$ & $15(46.9 \%)$ & $0.334^{\mathrm{b}}$ \\
\hline ICU duration (days) & $5.69( \pm 4.27)$ & $3.53( \pm 2.85)$ & $0.970^{\mathrm{b}}$ \\
\hline
\end{tabular}




\section{Cureus}

\begin{tabular}{|c|c|c|c|}
\hline \multicolumn{4}{|l|}{ Nephrotoxic medications } \\
\hline Aminoglycosides & $16(50 \%)$ & $8(25 \%)$ & $0.737^{\mathrm{b}}$ \\
\hline NSAIDS & $6(18.8 \%)$ & $4(12.5 \%)$ & $0.265^{b}$ \\
\hline Loop diuretic & $20(62.5 \%)$ & $23(71.9 \%)$ & $0.802^{b}$ \\
\hline Amphotericin B & $2(6.3 \%)$ & $1(3.1 \%)$ & $0.923^{b}$ \\
\hline Acyclovir & $0(0 \%)$ & $1(3.1 \%)$ & $1.000^{b}$ \\
\hline ARB & $5(15.6 \%)$ & $6(18.8 \%)$ & $0.974^{b}$ \\
\hline Contrast media & $7(21.9 \%)$ & $3(9.4 \%)$ & $0.319^{b}$ \\
\hline Vasopressor agents & $6(18.8 \%)$ & $11(34.4 \%)$ & $0.949^{b}$ \\
\hline Chemotherapeutic agents & $1(3.1 \%)$ & $1(3.1 \%)$ & $0.999^{b}$ \\
\hline Number of concomitant nephrotoxins & $1.97( \pm 1.09)$ & $1.81( \pm 1.06)$ & $0.905^{b}$ \\
\hline VAN trough & $12.20( \pm 4.84)$ & $11.35( \pm 4.79)$ & $0.158^{b}$ \\
\hline
\end{tabular}

\section{TABLE 1: Comparison of Clinical Characteristics in Matched Patients}

a Values shown are mean \pm standard deviation or number + percentage.

${ }^{\mathrm{b}}$ Binary logistic regression (the association between variables and AKI)

ARB: angiotensin II receptor blockers; CHD: congenital heart disease; CNS disease: central nervous system disease; GU/GI: genitourinary/gastrointestinal; ICU: intensive care unit; NSAIDS: nonsteroidal anti-inflammatory drugs; PTZ: piperacillin-tazobactam; SSTI: skin and soft tissue infection; VAN: vancomycin

As seen in Table 1, the groups were well-balanced in terms of age, gender, weight, ICU duration, concomitant administration of nephrotoxic agents, chronic disease, sepsis, and baseline creatinine.

Because sepsis contributes to the administration of multi-antibiotic combination, we included it in our matching criteria with a total number of 12 of 32 (37.5\%) in the VAN-PTZ group and for the other group, the total numbers were 11 out of 32 (34.4\%). The baseline creatinine in the VAN-cefotaxime group was higher than that in the VAN-PTZ group, although the association between the baseline creatinine and AKI was insignificant $(\mathrm{P}=0.065)$.

\section{AKI development in both treatment groups}

AKI was noted in 10 out of 32 patients (31.25\%) who were using VAN+ PTZ and in 13 out of 32 patients (40.62\%) who were using cefotaxime+ VAN, although there was no statistical significance between the two groups (p-value $=0.602$ ) $($ Table 2$)$. Most patients who were on VAN-PTZ regimen developed AKI Stage 1 (8/10, 80\%), while most patients who were using cefotaxime + VAN developed AKI Stage 2 (6/13, 46\%). No statistical significance was noted in all stages (Table 3).

\begin{tabular}{|c|c|c|c|}
\hline \multicolumn{2}{|c|}{ Characteristics } & VAN-PTZ & VAN-cefotaxime \\
\hline \multirow{2}{*}{ AKI } & Yes & $10(31.25 \%)$ & $13(40.62 \%)$ \\
\hline & No & $22(68.75 \%)$ & $19(59.37 \%)$ \\
\hline \multicolumn{2}{|l|}{ Total } & 32 & 32 \\
\hline
\end{tabular}

\section{TABLE 2: AKI Development in Both Treatment Groups}

AKI: acute kidney injury; PTZ: piperacillin-tazobactam; VAN: vancomycin 


\section{Cureus}

\begin{tabular}{|c|c|c|c|c|}
\hline Characteristics & VAN-PTZ $(n=32)^{a}$ & VAN-cefotaxime $(n=32)^{a}$ & Total $(n=32)^{a}$ & P-value \\
\hline Stage 1 & $8(61.5 \%)$ & $5(38.4 \%)$ & $13(20.3 \%)$ & 0.534 \\
\hline Stage 2 & $1(3.1 \%)$ & $6(18.8 \%)$ & $7(10.9 \%)$ & 0.109 \\
\hline Stage 3 & 1 (3.1\%) & $2(6.3 \%)$ & $3(4.6 \%)$ & 1.000 \\
\hline
\end{tabular}

\section{TABLE 3: AKI in Different Stages}

a Values shown are numbers and percentages.

AKI: acute kidney injury; PTZ: piperacillin-tazobactam; VAN: vancomycin

\section{Discussion}

The purpose of our study was to detect the incidence of AKI during treatment with VAN-PTZ or VAN + cefotaxime combination in pediatric age groups. In this study, the hospitalized children who were exposed to $\mathrm{VAN}+$ cefotaxime were at a greater risk of AKI than were children exposed to the VAN-PTZ treatment combination (40.6\% vs 31.3\%). To our knowledge, this is the first matched cohort study showing a difference in the incidence of AKI among diverse pediatric populations treated with this antibiotic combination.

Previous studies investigated the nephrotoxicity of those two combinations by choosing one drug (cefepime) from the cephalosporin class as an example for the entire group [17, 20], but our results were inconsistent with theirs. Cook et al. evaluated the incidence of AKI development in VAN-PTZ vs VAN + cefepime in the pediatric population (VAN-PTZ 28.9\% vs VAN + cefepime 7.9\%, p-value $=0.001$ ) [19]. Another unmatched study by Gomes et al. in 2014 showed that the VAN-PTZ combination caused AKI more frequently than the VAN + cefepime combination in adults (VAN-PTZ 34.8\% vs VAN + cefepime $12.5 \%$, p-value $=0.0001)$ [17].

Furthermore, a study by Al Yami on adults observed the relation between VAN with PTZ or with meropenem but did not reveal significant results between the two groups with respect to AKI development (VAN-PTZ 7.41\% vs VAN + meropenem 5.33\%, $\mathrm{P}=0.4)[12]$.

In our study, AKI was noted in $31.3 \%$ of the subjects in the VAN-PTZ group vs. $40.6 \%$ in the VAN + cefotaxime group. The results were statistically insignificant ( $p$-value $=0.602$ ). Most patients who used the VAN-PTZ regimen developed Stage 1 AKI (8/10, 80\%), whereas most VAN + cefotaxime patients developed Stage 2 AKI (6/13, 46\%); again, no statistical significance was noticed in all AKI stages. This shows that VAN + cefotaxime is as nephrotoxic as VAN-PTZ; therefore, the regimen should be used with caution, especially in children with underlying kidney disease. However, the mechanism of its nephrotoxicity is not fully understood. Additional studies are needed to validate this hypothesis. Resolution or long-term sequelae from AKI are uncertain. Data were not collected.

This study has several limitations. First, some data were difficult to obtain from the database and were taken manually (therapy duration and baseline and daily serum creatinine). Some data were missing (adjusted weighted VAN). The second issue was that this is a single-center study.

\section{Conclusions}

In conclusion, our study showed that cefotaxime + VAN is as nephrotoxic as VAN-PTZ. The mechanism is not understood. Since our study is the first to focus on cefotaxime + VAN, we believe that a multicentric study is necessary to confirm our results. After confirmation, we recommend decreasing the use of the VAN + cefotaxime regimen, especially in neonates, considering their immature kidneys.

\section{Additional Information \\ Disclosures}

Human subjects: Consent was obtained by all participants in this study. Institutional Review Board (IRB) of the King Abdulaziz University Hospital (KAUH) Jeddah, Saudi Arabia issued approval HA-020-J-008. Animal subjects: All authors have confirmed that this study did not involve animal subjects or tissue. Conflicts of interest: In compliance with the ICMJE uniform disclosure form, all authors declare the following: Payment/services info: All authors have declared that no financial support was received from any organization for the submitted work. Financial relationships: All authors have declared that they have no financial relationships at present or within the previous three years with any organizations that might have an interest in the submitted work. Other relationships: All authors have declared that there are no other relationships or activities that could appear to have influenced the submitted work. 


\section{Acknowledgements}

We would like to thank the Road of Change team for their constant support. We also express our gratitude to Dr. Abeer Najjar, Pediatric Infectious Diseases Consultant at King Abdulaziz University Hospital for comments that greatly improved the manuscript.

\section{References}

1. Kim SY, Moon A: Drug-induced nephrotoxicity and its biomarkers. Biomol Ther (Seoul). 2012, 20:268-72. 10.4062/biomolther.2012.20.3.268

2. Bruniera FR, Ferreira FM, Saviolli LR, et al.: The use of vancomycin with its therapeutic and adverse effects: a review. Eur Rev Med Pharmacol Sci. 2015, 19:694-700.

3. Makris K, Spanou L: Acute kidney injury: definition, pathophysiology and clinical phenotypes . Clin Biochem Rev. 2016, 37:85-98

4. Bellomo R, Ronco C, Kellum JA, Mehta ML, Palevsky P, the ADQI workgroup: Acute renal failure: definition, outcome measures, animal models, fluid therapy and information technology needs: the second international consensus conference of the acute dialysis quality initiative (ADQI) group. Crit Care. 2004, 8:R204-12. 10.1186/cc2872

5. Mehta RL, Kellum JA, Shah SV, et al.: Acute Kidney Injury Network: report of an initiative to improve outcomes in acute kidney injury. Crit Care. 2007, 11:R31. 10.1186/cc5713

6. KDIGO Clinical Practice Guideline for Acute Kidney Injury. (2012). Accessed: January 26, 2020: http://kdigo.org/wp-content/uploads/2016/10/KDIGO-2012-AKI-Guideline-English.pdf.

7. Elyasi S, Khalili H, Dashti-Khavidaki S, Mohammadpour A: Vancomycin-induced nephrotoxicity: mechanism, incidence, risk factors and special populations. A literature review. Eur J Clin Pharmacol. 2012, 68:1243-55. 10.1007/s00228-012-1259-9

8. Bentley ML, Corwin HL, Dasta J: Drug-induced acute kidney injury in the critically ill adult: recognition and prevention strategies. Crit Care Med. 2010, 38:169-74. 10.1097/CCM.0b013e3181de0c60

9. Tune BM: Nephrotoxicity of beta-lactam antibiotics: mechanisms and strategies for prevention . Pediatr Nephrol. 1997, 11:768-72. 10.1007/s004670050386

10. Burgess LD, Drew RH: Comparison of the incidence of vancomycin-induced nephrotoxicity in hospitalized patients with and without concomitant piperacillin-tazobactam. Pharmacotherapy. 2014, 34:670-76. 10.1002/phar.1442

11. Le J, Nguyen T, Law AV, Hodding J: Adverse drug reactions among children over a 10-year period. Pediatrics. 2006, 118:555-62. 10.1542/peds.2005-2429

12. Al Yami MS: Comparison of the incidence of acute kidney injury during treatment with vancomycin in combination with piperacillin-tazobactam or with meropenem. J Infect Pub Health. 2017, 10:770-73. 10.1016/j.jiph.2016.11.007

13. WHO Model Prescribing Information: Drugs used in Bacterial Infections: Cefotaxime . (2001). Accessed: July 16, 2018: http://apps.who.int/medicinedocs/en/d/Js5406e/16.9.html\#Js5406e.16.9.

14. Dilworth TJ, Ibrahim O, Hall P, Sliwinski J, Walraven C, Mercier RC: $\beta$-lactams enhance vancomycin activity against methicillin-resistant Staphylococcus aureus bacteremia compared to vancomcyin alone. Antimicrob Agents Chemother. 2014, 58:102-109. 10.1128/AAC.01204-13

15. Davies SW, Efird JT, Guidry CA, Dietch ZC, Willis RN, Shah PM, Sawyer RG: Top guns: the "Maverick" and “Goose” of empiric therapy. Surg Infect (Larchmt). 2016, 17:38-47. 10.1089/sur.2015.104

16. Moenster RP, Linneman TW, Finnegan PM, Hand S, Thomas Z, McDonald JR: Acute renal failure associated with vancomycin and $\beta$-lactams for the treatment of osteomyelitis in diabetics: piperacillin-tazobactam as compared with cefepime. Clin Microbiol Infect. 2014, 20:0384-89. 10.1111/1469-0691.12410

17. Gomes DM, Smotherman C, Birch A, Dupree L, Della Vecchia BJ, Kraemer DF, Jankowski CA: Comparison of acute kidney injury during treatment with vancomycin in combination with piperacillin-tazobactam or cefepime. Pharmacotherapy. 2014, 34:662-69. 10.1002/phar.1428

18. Rutter WC, Burgess DR, Talbert JC, Burgess DS: Acute kidney injury in patients treated with vancomycin and piperacillin-tazobactam: a retrospective cohort analysis. J Hosp Med. 2017, 12:77-82. 10.12788/jhm.2684

19. Cook KM, Gillon J, Grisso AG, Banerjee R, Jimenez-Truque N, Phillips EJ, Van Driest SL: Incidence of nephrotoxicity among pediatric patients receiving vancomycin with either piperacillin-tazobactam or cefepime: A cohort study. J Pediatric Infect Dis Soc. 2019, 8:221-27. 10.1093/jpids/piy030

20. Hammond DA, Smith MN, Painter JT, Meena NK, Lusardi K: Comparative incidence of acute kidney injury in critically ill patients receiving vancomycin with concomitant piperacillin-tazobactam or cefepime: a retrospective cohort study. Pharmacotherapy. 2016, 36:463-71. 10.1002/phar.1738 